\title{
CRISE DE SIGNE Le symbole dans le théâtre de Maeterlinck Gérard Dessons²
}

" Il me semble que la pièce de théâtre doit être avant tout un poème. » MAETERLINCK (1990 : 155), propos rapporté par Jules Huret (1893).

« La porte symbolise le lieu de passage entre deux états, entre deux mondes, entre le connu et l'inconnu, la lumière et les ténèbres, le trésor et le dénuement » CHEVALIER et GHEERBRANT (1982: 779).

RÉSUMÉ : L'œuvre de Maurice Maeterlinck présente cette caractéristique paradoxale d'être considérée comme l'une des plus importantes du mouvement symboliste alors que, dans le même temps, elle participe à l'invention d'un type de symbole - le symbole de l'œuvre - qui se révèle critique du symbolisme en général. Contrairement au symbole du dictionnaire, qui fonde sa valeur sur l'universalité, le symbole de l'œuvre tire sa valeur de sa spécificité. Ainsi, dans La Mort de Tintagiles, la porte, objet symbolique par excellence, perd sa valeur universelle de passage entre deux mondes, pour gagner une fonction : faire du langage, dans son phrasé même, le lieu dramatique d'une communication impossible.

MOTS-CLÉS : Maeterlinck ; Poétique ; Signe ; Symbole ; Théâtre.

\footnotetext{
${ }^{1}$ Publié originalement dans Coulisses, Besançon, Théâtre universitaire-Presses universitaires de Franche-Comté, n 32 , mai 2005, p. 49-61.

${ }^{2}$ Gérard Dessons est professeur de langue et littérature françaises à l'Université Paris 8 et a consacré de nombreux travaux à la poétique, à la théorie du langage et à la théorie de l'art. Il a notamment publié Maeterlinck, le théâtre du poème (2005, réed. 2016), Benveniste ou l'invention du discours (2006), Rembrandt ou l'odeur de la peinture (2006), L'Art et la manière (20049, La Manière folle (2010), Le Poème (2011) et La Voix juste. Essai sur le bref (2015).
} 


\section{CRISE DE SIGNO. O SÍMBOLO NO TEATRO DE MAETERLINCK}

RESUMO: A obra de Maurice Maeterlinck apresenta a característica paradoxal de ser considerada como uma das mais importantes do movimento simbolistas enquanto, ao mesmo tempo, ela participa da invenção de um tipo de símbolo - o símbolo da obra - que se revela crítico do simbolismo em geral. Contrariamente ao símbolo do dicionário, que fundamenta seu valor na universalidade, o símbolo da obra tira seu valor de sua especificidade. Assim, em A Morte de Tintagiles, a porta, objeto simbólico por excelência, perde seu valor universal de passagem entre dois mundos, para ganhar uma função: fazer da linguagem, em seu próprio fraseado, o lugar dramático de uma comunicação impossível.

PALAVRAS-CHAVE: Maeterlinck; Poética; Signo; Símbolo; Teatro.

La position de Maeterlinck vis-à-vis de la littérature et du langage est, à l'image de nombreux écrivains associés au symbolisme, ambiguë. Imprégné de métaphysique et de mysticisme (sa lecture et traduction de L'Ornement des noces spirituelles de Ruysbroeck l'Admirable - dédicataire d'À rebours de Huysmans [1884] - est pour lui une révélation, qu'il prolonge avec la traduction des Disciples à Sais et des Fragments de Novalis), il travaille à un théâtre d'où sortiront les dramaturgies modernes. L'essai Le Trésor des humbles (1896) est de ce point de vue un livre complexe. Sa tonalité générale est mystique : il comprend notamment une étude sur Ruysbroeck, un extrait de sa préface à sa traduction de Novalis, une étude sur Emerson, et des textes sur «La morale mystique », «Le réveil de l'âme », « La vie profonde » et «Le silence ». Mais les propos qu'il contient sur le théâtre et sur le langage, même s'ils s'inscrivent dans la tonalité générale de l'ouvrage, ouvrent des perspectives tout autres, que vérifie sa pratique de poète dramatique.

Un texte comme «Le silence », par exemple, est directement issu de la pensée mystique. Il désigne le commerce des âmes, opposé à la communication par le langage, qui est le commerce des hommes. Chaque petit silence quotidien y est hanté par le « grand silence ${ }^{3}$ final. Cependant, ce texte propose une réflexion sur les rapports entre langage et silence que bien des conceptions philosophiques et linguistiques actuelles n'ont pas encore commencé de penser. Notamment la distinction entre silence actif et silence passif, et, surtout, l'approche positive du silence, qui n'est pas considéré ici comme une simple absence de paroles ${ }^{4}$. Le silence, alors, peut devenir, comme chez Mallarmé, une catégorie du langage à part entière.

\footnotetext{
3 Je fais allusion au livre Avant le grand silence (MAETERLINCK, 1934).

4 "Les paroles que nous prononçons n'ont de sens que grâce au silence où elles baignent " «Le silence » (MAETERLINCK, 1998: 22)
} 
Le symbolisme s'est construit sur le terreau mystique, voire ésotérique, de l'époque, orientant le symbole dans une direction métaphysique. Historiquement, le symbole a précédé le symbolisme ${ }^{5}$, au sens où, même si les mots symbole et symbolisme ont certainement cohabité, le symbolisme en tant qu'école littéraire, avec les influences idéalistes, mystiques et métaphysiques qu'il a subies, a succédé au symbole comme problématique de la signification, effaçant souvent les enjeux de départ par une contextualisation a posteriori de cette interrogation.

La question du symbole - partiellement prise en charge par le symbolisme (c'est pourquoi il est préférable de parler des symbolismes) - participe d'une interrogation historique sur le langage et, via la littérature, sur l'art en général. Cette réflexion va provoquer, au sein du langage, une crise du signe et du logos, alors même que le symbole relève, du point de vue de son fonctionnement, de la logique générale du signe: un représentant tient lieu d'un représenté ${ }^{6}$. Ainsi, toute l'époque travaille sur la suggestion et l'évocation ${ }^{7}$, opposées à la nomination, à l'expression, à partir d'une problématisation - et donc d'une historicisation - de l'ineffable et de l'inexprimable mystiques ${ }^{8}$. L'époque est critique et inventive. Ses objectifs sont les suivants : " recherche des nuances, recul des homes de la langue, expression d'idées neuves avec des formes nouvelles et des mots qu'on n'a pas entendus encore $"$ ". C'est en effet une autre conception du langage que les écrivains s'emploient à élaborer. De nouvelles catégories se mettent en place, opératoires aussi bien pour la littérature que pour l'étude du langage, comme le rythme « immanent aux mouvements mêmes de la pensée » (GHIL, 1978:162) -, le silence, la musique.

Pour Maeterlinck, la véritable question littéraire et théâtrale est celle du symbole. Ses propos sur le symbole, dans sa « Réponse à l'enquête de Jules Huret » de 1891, sont en réalité une réflexion sur la littérature et l'œuvre d'art. Ainsi, la distinction qu'il opère entre le symbole «a priori », « de propos délibéré », et le symbole "inconscient », se produisant « à l'insu du poète », ouvre sur une critique implicite de ce symbolisme - alors ambiant qui conçoit une œuvre comme la réalisation singulière d'un symbole éternel, c'est-à-dire comme une allégorie : « Je ne crois pas que l'œuvre puisse naître viablement du symbole; mais le symbole

\footnotetext{
5 « En 1885 [...] nous parlions de symbole, nous n'avions pas créé le mot générique de symbolisme » (KAHN, 1936: 33). Cité dans Les Théories poétiques à lépoque symboliste (1883-1896) (BIÉTRY, 2001: 50).

6 "Si l'on donne au mot «signe» un sens générique par où il englobe celui de symbole (qui dès lors le spécifie), on peut dire que les études sur le symbole relèvent de la théorie générale des signes, ou sémiotique » (TODOROV, $1977: 9$ ).

7 «Symboliser est évoquer, non dire et narrer et peindre » (René Ghil, 1885, qui parle aussi du « réel et suggestif Symbole ») ; «La Suggestion peut ce que ne pourrait l'expression. La Suggestion est le langage des correspondances. " (Charles Morice, 1889) ; "Nommer un objet, c'est supprimer les trois quarts de la jouissance du poème qui est faite de deviner peu à peu : le suggérer, voilà le rêve. " (Mallarmé, 1891)

${ }^{8}$ Depuis $A$ rebours, de Huysmans (1884), c'était devenu un topos d'époque: «Nous rendons l'impossible, nous exprimons l'inexprimable » (FLOUPETTE, $1972: 53)$. Cité dans Biétry (2001:51). ${ }^{9}$ Henri de Régnier, revenant, dans une conférence donnée le 6 février 1900, sur ce moment littéraire. Cité dans Roland Biétry (2001).
} 
naît toujours de l'œuvre si celle-ci est viable » (MAETERLINCK, 1999: 586) ${ }^{10}$. Davantage qu'une appartenance d'époque au symbolisme, il y a surtout, chez Maeterlinck, une attention à la question du symbolique. Au lieu de partir du symbole (en tant que signe universel) pour aller vers l'œuvre, qui en réaliserait alors une application particulière, Maeterlinck part de l'œuvre, de son écoute, pour entendre ce qu'elle fait, ce qu'elle signifie en tant que dire symbolique. Le symbole devient alors le nom de l'inconnu de l'œuvre.

Le poème dramatique constitue, pour Maeterlinck, le meilleur champ de recherche sur le langage, parce qu'il permet de travailler, à travers le dialogue, sur l'empirique du conversationnel, et, à travers le rapport entre langage et réalité, sur la question de la référence. Il va s'agir, pour lui, de mettre sur le devant de la scène ce que es tragiques avaient seulement donné à sous-entendre. Faire entendre le dire à travers ce qui est dit : " Si je vous parle en ce moment des choses les plus graves, de l'amour, de la mort ou de la destinée, je n'atteins pas la mort, l'amour ou le destin, et malgré mes efforts, il restera toujours entre nous une vérité qui n'est pas dite, qu'on n'a même pas idée de dire » (MAETERLINCK, 1998 : 21). Et du point de vue du symbole, de cet inconnu de l'œuvre qui invente ce qu'elle signifie, la mort, l'amour, le destin, ne peuvent pas constituer des thèmes dont on parle, mais des vérités éthiques qu'il faut dire dans l'invention sans cesse renouvelée des œuvres de langage. On peut penser au propos de Valère Novarina : « Ce dont on ne peut parler, c'est cela qu'il faut dire » (1999: 29), qui retourne la maxime bien connue de Wittgenstein : "Ce dont on ne peut parler, il faut le taire » $(1961: \text { 177 })^{11}$. Simplement, ce qu'il faut dire est à dire autrement que comme propos, thème, énoncé, message. Il faut le dire comme phrasé.

\section{LE LIEU SCENIQUE EST DANS LE LANGAGE}

Ce conflit entre le dit et le dire, l'énoncé et le phrasé, le signe et le rythme, dont le symbole est le lieu théorique, le théâtre en constitue, pour Maeterlinck, le lieu poétique. Un lieu tout à la fois poétique et critique, dans la logique du « poème critique » de Mallarmé : poétique parce que critique, critique parce que poétique.

Pour rendre compte de cette mise en crise du signe et de sa logique, je me propose de réfléchir sur la question de la référence et de la représentation, spécifiquement dans le rapport du langage et du lieu scénique, question qui se traduit, généralement, dans la mise en scène, par un traitement particulier du décor.

On connait les principes du théâtre réaliste concernant le décor et les accessoires. L’idée du quatrième mur d'Antoine transforme la scène en véritable échantillon du réel. Les objets sont identiques à ceux que le spectateur utilise quotidiennement.

\footnotetext{
10 «Réponse à l'enquête de Jules Huret », 1891.

11 «Wowon man nicht sprecher kann, daruber muss mon schnweigen. » - Traduction de GillesGaston Grangier: «Sur ce dont on ne peut parler, il faut garder le silence » $(1993: 177)$.
} 
Cette conception est aussi - et, s'agissant de théâtre, d'abord - une conception du langage. Les mots y sont compris comme des désignateurs de choses. Selon ce point de vue, dans La Princesse Maleine, le manteau rouge de la reine, "ce manteau rouge » (MAETERLINCK, $1998: 135)^{12}$, qui se trouve « ici » (ibid.), devrait être, sur la scène comme dans la vie, un véritable manteau, et il devrait être rouge, le monde se trouvant alors en totale redondance avec le langage conçu, selon le mot de Saussure, comme une nomenclature. D'un point de vue linguistique, cette conception repose sur la confusion du signifié du signe avec l'éventuel référent du discours (la «fonction référentielle » [referential function] de Jakobson).

Cette confiance dans le pouvoir désignateur des mots se trouve mise à l'épreuve par l'écriture de Maeterlinck, ne serait-ce qu'au simple plan de l'énoncé, lorsque les apparentes contradictions, si elles ne sont pas attribuées, réalistement, à la psychologie des personnages, mettent en doute cette fonction désignative : «Pourquoi n’y a-il pas de lumière, sœur Ygraine ? / / Il y en a, mon enfant » (22) ; ou encore : «il ne faut pas qu'un oiseau ou une herbe nous entende » / « Il n'y a pas d'herbe petite sœur » (12). Ce dernier exemple met bien en évidence le conflit généré par l'écriture entre la lecture réaliste de l'énoncé et l'attribution à celui-ci d'une valeur gnomique ( «il ne faut pas qu'un [seul] oiseau ou une [seule] herbe nous entende »). Le problème est celui d'une situation par rapport au langage. Qu'est-ce que dire qu'est-ce qui est dit dans le dire ? Et, questions liées : qu'est-ce qu'entendre ? qu'est-ce qui est à entendre dans ce qui est dit ?

Cette série d'interrogations, un metteur en scène peut se les poser lorsqu'il a à réfléchir sur le décor d'une pièce, c'est-à-dire sur la nature du lieu dramatique. Ce problème, le théâtre de Maeterlinck, comme, plus généralement, le théâtre symboliste, le pose inévitablement. Il est contenu dans cette réplique d'Ygraine, dans $L a$ Mort de Tintagiles : «(Regardant autour d'elle.) Je n'avais jamais vu tout ceci » (1997: 35, Acte V). Le déictique ceci fait coïncider, en tant que lieu dramatique, le monde du théâtre et le monde du langage. Ce qui est impliqué dans ceci, le texte ne nous le dit pas précisément. Il fait juste allusion à une impossibilité de mouvement et à quelques impressions : " On ne peut pas monter plus haut ; et tout est défendu... Il fait froid. .. Il fait si noir aussi qu'on aurait peur de respirer... » (ibid.). C'est que l'extériorité du À lieu est tout entier contenu dans le regard d'une subjectivité parlante. Une seule chose, parmi « tout ceci », se trouve décrite : une porte. «Il y a là une porte effrayante... » (ibid.). Ygraine est seule en scène. Son interlocuteur, destinataire logique de la désignation, est donc, sur le plan de la fiction, elle-même ; mais sur le plan dramaturgique, il est le public-lecteur. En fait, la « description » d'Ygraine ne nous dit pas ce qu'elle voit « réalistement », mais ce qu'il y a à voir. Et ce voir est un entendre. Il n'y a pas de porte qui ne soit ici ce qui en est dit : «Oh ! elle est froide !... Elle est en fer uni ; tout uni et n'a pas de serrure. .. Par où donc s'ouvre-t-elle ? Je ne vois pas de gonds... Je crois qu'elle est scellée dans la muraille... » (ibid.). Le décor est celui que la parole met en place.

12 « HJALMAR - Comment ce manteau rouge est-il ici ?». 
Maeterlinck était justifié, alors, de demander à Lugné-Poe, pour la mise en scène de Pelléas, "deux décors d'imprécision, deux sortes de toiles de fond "d'accompagnement" » (DOCQUOIS, $1990: 159)$. On sait que lors de la première représentation de Pelléas au théâtre de L'Euvre, la salle était séparée de la scène par un voile de gaze, qui organisait un brouillard de la vision.

Cette porte, que La Mort de Tintagiles donne à entendre-voir, je propose de m'y intéresser comme à l'un des « objets » les plus importants du théâtre de Maeterlinck, d'autant plus importants que, à l'image de l'anneau dans Pelléas, ils sont peu nombreux, et qu'ils ont donc tendance à focaliser sur eux les regards et les paroles. La porte est en scène dès l'ouverture de Pelléas (I, I) ; elle est au centre de La Princesse Maleine (II, 5 ; IV, 5) ; elle clôt le drame de Tintagiles (V).

\section{LA PORTE DE TINTAGILES}

C'est précisément son statut dans La Mort de Tintagiles que je voudrais examiner. Sur le plan du récit, on se souvient que le petit Tintagiles a été enlevé par les servantes de la reine, et que la fin de la pièce voit l'enfant et sa sœur Ygraine séparés par la porte qui ferme la tour où vit la reine-ogresse. Est en question ici le lieu du drame. Tintagiles et Ygraine cherchent à se rejoindre à travers une porte qui les en empêche. Cette porte fonctionne comme une frontière entre le monde d'ici - «Qui est-ce qui t’a fait venir ici ? » (p. 10), « du temps qu'il y avait ici des hommes » (p. 18) -, celui du visible, où vivent Tintagiles, ses sœurs et leur serviteur Aglovale; et un monde de là-bas, celui de l'invisible reine : « c'est là que se trouve le trône de la reine » (p. 12), « elle vit là, toute seule dans sa tour » (p. 13), « elle est là depuis des années dans son énorme tour, à dévorer les nôtres » (p. 18).

Mais cette porte, bien qu'elle soit nommée, n'a pas à être représentée sur la scène en tant qu'objet. Elle se trouve matérialisée en tant que seuil et lieu de passage interdit, par le seul discours des personnages. Non en ce qu'elle y est abondamment nommée, mais en ce que le langage, qui est un instanciateur éthique, c'est-à-dire un marqueur de positions, la figure subjectivement au gré du dialogue. Ainsi, les interlocuteurs de la scène n'ont de cesse de construire des repérages par le langage, qui organise leurs relations par rapport à une porte qui se trouve n'exister qu'en fonction de ces mouvements interlocutifs, indépendamment de la situation physique des personnages. Ainsi, Ygraine alterne les positions de parole, s'adressant à Tintagiles indifféremment à partir de son ici de parole à elle (lui se trouve donc là) : « Où es-tu ?... es-tu là ? » (p. 36), ou à partir de l'ici de parole de Tintagiles (c'est elle, alors, qui se trouve là) : « N’aie pas peur, je suis là... » (p. 37).

Ces positions sont des stratégies subjectives inconscientes. Ainsi, Ygraine, qui frappe du poing et des pieds contre la porte, invective la reine à partir d'un ici de sa parole qui ne coïncide pas avec le lieu où elle se trouve physiquement en tant que personnage : «Oh! le monstre ! le monstre !... C'est ici que vous êtes !... » (p. 35). 
C'est qu'elle vient de passer discursivement de l'autre côté du seuil, en englobant la reine dans l'ici de sa parole. Symétriquement, Tintagiles, qui se trouve pourtant du même côté de la porte que la reine, situe la présence de celle-ci à l'adresse d'Ygraine, avec le marqueur là : « Elle est là !... » (p. 38), repérant la reine non par rapport à sa position physique, mais par rapport à l'ici de la parole d'Ygraine. Ce faisant, l'ici d'Ygraine devient le sien. Tintagiles, à son tour, vient de passer, discursivement, de l'autre côté de la porte, afin de rejoindre Ygraine. Deux répliques plus loin, le lieu de référence de Tintagiles redevient l'ici de sa parole : «Oh ! oh ! sœur Ygraine, viens ici... » (ibid.). La porte, qui est l'organisateur symbolique de ce ping-pong discursif, est bien autre chose qu'une chose, qu'un objet, voire qu'un symbole universel (les portes de corne et d'ivoire des hermétistes) ${ }^{13}$. Elle est une modalité discursive. C'est en cela que le décor est dans le langage.

Dans ce dernier acte de la pièce, la porte joue un rôle capital en tant que symbole produit par l'œuvre. Elle est d'ailleurs elle-même, en tant qu'objet, le champ de cette tension entre l'union et la séparation discursives des personnages, et par deux fois. Tout d'abord, Tintagiles y décèle une fente à travers laquelle il perçoit la clarté qui entoure Ygraine : «Mais si ; je vois bien ta lumière... » (p. 37). Dans cette séquence, afin de se rejoindre par le regard, chaque personnage, alternativement, invite l'autre à voir à partir de son ici de parole :

\section{YGRAINE - De quel côté ? ici ?... dis, dis... c'est peut-être par là? \\ TINTAGILES - Ici, ici... Tu n'entends pas ? Je frappe... YGRAINE - Ici ?}

Chaque ici est à la fois un désir de réunion et son impossibilité. Ces ici se veulent solidaires, ils ne sont que solitaires. Infiniment, ils invitent l'autre du dialogue à se fondre dans l'origine commune de la parole ; mais, interrogatifs - « Ici ? » -, ils portent en eux, avec la force d'une parole mythique, leur possible non-coïncidence collective. Le paradoxe (logique et linguistique), c'est que cette transformation de la valeur éthique - de collective en individuelle - de la déixis se fait dans la coénonciation même du discours. En cela, la porte qui organise symboliquement ces échanges de paroles se révèle un ressort éminemment tragique.

La seconde occurrence où la porte est ce lieu dramatique qui n'existe que d'être fantasmé dans les discours des protagonistes est le moment où les deux personnages tentent de s'embrasser malgré l'obstacle qui les sépare :

YGRAINE - Je vais t'aider... embrasse-moi... au travers de la porte... ici... ici...

13 « Je n'ai pu percer sans frémir ces portes d'ivoire ou de corne qui nous séparent du monde invisible. " (NERVAL, 1993: 695) - L'image vient de Virgile (Énéide, VI), reprise d’Homère (Odyssée, XIX). 
TINTAGILES (très faiblement) - Ici... ici... sœur Ygraine... YGRAINE - C'est ici, c'est ici que je donne des baisers, tu l'entends ? encore ! encore!

TINTAGILES (de plus en plus faiblement) - J'en donne aussi... ici... sœur Ygraine !... sœur Ygraine !... Oh !... (p. 39)

Les deux personnages sont repérés par le seul ici de leur parole. Ces ici qui, linguistiquement, les isolent dans la solitude de leur discours - puisque lici du contact rêvé est alternativement celui de chacun des deux personnages - en réalité organise prosodiquement leur réunion, leur fusion.

Si on néglige la didascalie «(très faiblement) », qui peut fonctionner comme une simple indication de régie (elle n'a pas l'opacité de l'avant-dernière didascalie de la pièce : «Un long silence inexorable »), la fin de la réplique d'Ygraine (ici... ici...), et le début de celle de Tintagiles (Ici... ici ...), se trouvent en relation de continuité par le hiatus qui les lie (ici-ici), et qui fait apparaître les paroles de Tintagiles comme un écho affaibli de celles d'Ygraine. Tout se passe comme si les deux personnages prenaient en charge une seule et même instance de parole, comme si, en même temps que les ici solipsistes, n'existait plus qu'un seul ici, celui, fusionne, du drame. Mais cet ici du drame tire sa valeur précisément de la tension qu'il réalise entre le discontinu des ici individuels et le continu prosodique qui les unit.

Un fonctionnement similaire se retrouve dans Pelléas et Mélisande, au moment où Golaud surprend Pelléas et Mélisande dans la scène de la chevelure (III, 2) :

$$
\begin{aligned}
& \text { GOLAUD - Que faites-vous ici ? } \\
& \text { PELLÉAS - Ce que je fais ici ?... Je... } \\
& \text { GOLAUD - Vous êtes des enfants... } \\
& \text { (MAETERLINCK, 1992: 38) }
\end{aligned}
$$

Le travail poétique a lieu dans ce cas non par un hiatus, mais par un effet de rime. Ici, lieu de la parole des deux personnages, devrait coïncider avec le lieu de la scène. Mais les deux personnages ne vivent pas la même situation. En demandant «Que faites-vous ici ? », Golaud s'adresse à Pelléas et Mélisande, qu'il vient de surprendre ensemble ( Vous êtes des enfants »). Mais la réponse de Pelléas, « Ce que je fais ici ?», montre qu'il a interprété le vous de Golaud comme s'adressant à lui seul (les deux frères se vouvoient).

De fait, sa réplique n'est pas une réponse, mais la reformulation de la première question. Le parallélisme des deux répliques introduit une tension entre les deux ici, alors, qui n'en font qu'un : l'ici du drame comme disjonction entre deux histoires. La réplique de Pelléas opère ainsi un escamotage de Mélisande (elle reste muette), en interprétant le vous comme le concernant seul. D'ailleurs, la fin de la réplique met en scène, en l'accentuant comme fin de groupe, le pronom je: « $\mathrm{Ce}$ que je fais ici ?... Je... » 
Pour revenir au statut de la porte dans La Mort de Tintagiles, en fait, le drame pose la question : qu'est-ce qu'une porte ? Question à laquelle on ne peut répondre qu'en lui substituant cette autre question : qu'est-ce qu'une porte dans La Mort de Tintagiles?

Il s'agit d'une porte inouie, invue, inventée par ce poème. Une porte qui excède sa fonctionnalité d'être à la fois passage et obstacle. Une porte éthique. Elle est lien et rupture, espoir et découragement. Sa réalité n'est pas matérielle, mais purement subjective. Intersubjective et transsubjective. La porte, cette porte, ne se trouve plus définie par le signe qu'elle est aussi (même si ce signe qu'elle ne peut pas ne plus être ne la spécifie pas), mais par le jeu des déictiques, morphèmes qui n'ont pas de signifiés (Benveniste parlait de signes vides), mais seulement une fonction, celle de rapporter l'expérience du monde à l'exercice du langage, qui est une activité impersonnelle parce que transpersonnelle. Ici est toujours l'ici d'un je, dont la référence empirique est nécessairement provisoire. C'est tout le drame de La Mort de Tintagiles : constater qu'entre les ici, des portes existent, ouvertes ou fermées, que ces ici soient pris en charge par un même personnage ou par des personnages différents.

Cette porte spécifique que le texte de Maeterlinck a inventée, est pourtant toujours une porte. C'est-à-dire une porte pour moi, qui, dans ma vie empirique, ai affaire aux portes, une porte qui me concerne dans ce que je ne connais pas - ou que je méconnais - des portes, mais qu'en tout cas je reconnais, assez pour, cette porte, me l'approprier en la co-énonçant en même temps que la voix plurielle et singulière des personnages.

C'est que cette porte est à la fois un signe et un symbole. En tant que signe, elle signifie à la manière d'un universel. C'est la porte des dictionnaires : une porte n'est pas un tiroir, ni une fenêtre. En tant que symbole, elle peut, bien entendu, également signifier à la manière d'un universel (dans un dictionnaire des symboles, par exemple $)^{14}$, c'est le symbole a priori que récuse Maeterlinck pour la littérature : un symbole qui signifie des valeurs anthropologiques à partir de la logique du signe. Mais la porte, en tant que symbole, peut aussi être inventée par l'œuvre, à la manière de ce symbole inconscient, inentendu jusqu'ici, que revendique Maeterlinck.

Dorénavant, en tant que lecteur, en tant que spectateur, dans mon rapport au signe « porte » existe quelque chose de neuf, qui n'est pas véritablement une signification supplémentaire, mais une dimension éthique : un sujet, une œuvre. Dans l'expérience du lecteur existe maintenant une « porte-Maeterlinck ». En cela, cette

\footnotetext{
${ }^{14}$ Voici deux autres extraits de l'entrée «Porte » du Dictionnaire des symboles de J. Chevalier et A. Gheerbrant, déjà cité (n. 2) : «La porte ouvre sur un mystère. Mais elle a une valeur dynamique, psychologique ; car non seulement elle indique un passage, mais elle invite à le franchir. C'est l'invitation au voyage vers un au-delà... / Le passage auquel elle invite est, le plus souvent, dans l'acception symbolique, du domaine profane au domaine sacré » (p. 779). « La porte a aussi une signification eschatologique. La porte comme lieu de passage, ct particulièrement d'arrivée, devient tout naturellement le symbole de l'imminence de l'accès et de la possibilité d'accès à une réalité supérieure (ou inversement de l'effusion de dons célestes sur la terre) » (p. 781).
} 
porte est indissociablement une idée et une manière. Une idée en tant qu'elle est une manière. Dans un texte sur la formation du goût par la lecture, Pierre Nicole, en 1671, évoquait cette question en parlant des «idées de manières et de tours » (Cf. NICOLE, 1992: 150) ${ }^{15}$ qui, par la fréquentation des œuvres, s’impriment dans l'esprit des lecteurs à la façon de «moules ou de cachets » (ibid). Pour cette raison, il conseillait de « lire quantité de bons vers, et n'en lire point de mauvais » (ibid.). Le point de vue est formulé à partir d'une métaphysique du beau, mais le principe reste fort : c'est l'œuvre qui fait l'idée, non le contraire.

« Je descends l'escalier sur la pointe des pieds et j'ouvre la porte comme si c'était une porte ordinaire... Mon Dieu ! Mon Dieu ! Qu'est-ce que je vois ! Devinez un peu ce que je vois !... » (MAETERLINCK, 1992 : 61). Cette réplique de la Vieille servante, dans Pelléas et Mélisande, fait figure d'allégorie du symbolique chez Maeterlinck. Dans ce théâtre, l'ordinaire se trouve mis en question. Non par sa récusation, mais par son interrogation. Il existe bien un extraordinaire, mais il n'est que l'autre côté de l'ordinaire. Au sens où l'ordinaire est toujours extraordinaire pour peu qu'on se mette à son écoute. Mais aussi l'extraordinaire ne me touche que parce qu'il se révèle être mon ordinaire. Toute cette violence du drame de Maeterlinck m'est, finalement, ordinaire, et quotidienne ${ }^{16}$. Finalement, parce qu'il a fallu que le poème ait lieu pour que j'aie ce savoir. Tout l'inconnu que la pièce donne à connaître comme un reconnaître se tient dans le «comme si » de la réplique: «J'ouvre la porte comme si c'était une porte ordinaire ». C'est-à-dire que je sais par avance que ce ne l'est pas. Ce que c'est, exactement, je ne sais. Mais cela : le fait que je ne sache quoi, je le sais. C'est même, poétiquement, mon seul savoir.

Car rien de positif n'est plus su quand le dire se libère du dit, tant le poème excède infiniment, en tant que symbole, les signes qu'il met en scène.

\section{RÉFÉRENCES BIBLIOGRAPHIQUES}

BIÉTRY, Roland. Les Théories poétiques à l'époque symboliste (1883-1896). Genève : Slatkine Reprints, 2001.

CHEVAlIER, Jean; GHEERBRANT, Alain. Dictionnaire des symboles. Mythes, rêves, coutumes, gestes, formes, figures, couleurs, nombres. Paris: Robert Laffont; Jupiter, 1982.

15 Voir Dessons (2004: 268).

${ }^{16}$ Le quotidien n'est pas l'ordinaire. Mais ces deux dimensions sont liées chez Maeterlinck, dans l'essai sur «Le tragique quotidien » notamment, où le dramaturge se propose de faire entendre, par-dessus les dialogues ordinaires de la raison et des sentiments, le dialogue plus solennel et ininterrompu de l'être et de sa destinée » (Le Trésor des humbles, 1998, p. 101). 
DESSONS, Gérard. L'Art et la manière. Art, littérature, langage. Paris: Champion, 2004.

DOCQUOIS, Georges. "Conversation avec Maurice Maeterlinck ». Le Journal, 17 mai 1893. In : MAETERLINCK, Maurice. Introduction à une psychologie des songes. Bruxelles : Labor, 1990.

FLOUPETTE, Adoré. Les Déliquescences. Milan : Cisalpino-Goliardica, 1972.

GHIL, René. Traité du verbe. Paris: Nizet, 1978.

HURET, Jules. «Conversation avec Maurice Maeterlinck». Le Figaro, 17 mai 1893.

KAHN, Gustave. Les Origines du symbolisme. Paris : Albert Messein, 1936.

MAETERLINCK, Maurice. Introduction à une psychologie des songes. Bruxelles : Labor, 1990.

. Avant le grand silence. Paris : Fasquelle, 1934.

. Le Trésor des humbles. Bruxelles : Éditions Labor, 1998.

. Euvres l. Le Réveil de l'âme. Poésie et essais. Édition établie et présentée par Paul Gorceix. Bruxelles : Éditions Complexe, 1999.

. La Princesse Maleine. Bruxelles: Éditions Labor, 1998.

. La Mort de Tintagiles. Paris : Répliques-Babel, 1997.

. Pelléas et Mélisande. Bruxelles: Éditions Labor, 1992.

NERVAL, Gérard de. Aurélia. In: Euvres complètes, Tome III. « La Pléiade». Paris : Gallimard, 1993.

NICOLE, Pierre. Prefácio ao Recueil de poésies chrétiennes et diverses. In : MORTGAT, Emmanuelle ; MÉCHOULAN, Éric (orgs.). Écrire au XVII ${ }^{e}$ siècle. Une anthologie. Textos escolhidos e apresentados por Emmanuelle Mortgat e Éric Méchoulan. Paris: Presses Pocket, 1992.

NOVARINA, Valère. Devant la parole. Paris: P.O.L., 1999.

TODOROV, Tzvetan. Théories du symbole. Collection Points. Paris: Seuil, 1977.

WITTGENSTEIN, Ludwig. Tractatus logico-philosophicus. Tradução de Pierre Klossowski. Paris: Gallimard, 1961. 